\title{
Piglets offer a peek at early brain development
}

Stress during early life can affect human cognitive development, but characterizing these effects is challenging, in part because of a lack of appropriate research subjects. A suitable model would be one whose brain development is similar to that of humans and whose cognitive abilities can be evaluated objectively. Rodney Johnson and his colleagues at University of Illinois at Urbana-Champaign recently developed a model that meets these requirements-the neonatal piglet.

First, they used magnetic resonance imaging (MRI) to quantify piglets' brain development from the ages of 2 weeks to 24 weeks, when the piglets were near sexual maturity. Total brain volume increased 121-130\% during this period. The piglets' brains reached about $50 \%$ of maximum volume at the age of 4 weeks and grew to about $95 \%$ of maximum volume by the age of 21-23 weeks (Dev. Neurosci. published online 6 July 2012; doi:10.1159.000339311). This large postnatal increase in piglet brain volume is similar to that of human neonates: at birth, the human brain is 25\% of its adult size, growing to $85-90 \%$ within the first 2 years of life.

Next, Johnson's team created a ' $T$ '-shaped maze task to evaluate piglets' learning. Two-week-old piglets were trained to locate a reward in a constant location within the maze by using visual cues placed outside the maze. Their performance improved over time, and they had mastered the task within $5 \mathrm{~d}$ (Anim. Cogn. 15, 667-676; 2012). The reward location was then changed, and piglets were tested again. Their performance was initially much worse, as expected, but improved over time. The results show that a T-maze task can be used to assess cognition in neonatal piglets.

But developing the T-maze task was itself a challenge. "[T]here were a lot of things that went wrong that we didn't predict," Johnson indicated in a press release. For example, the piglets ignored the food rewards that the researchers initially offered but were finally motivated to perform the maze task when the reward was changed

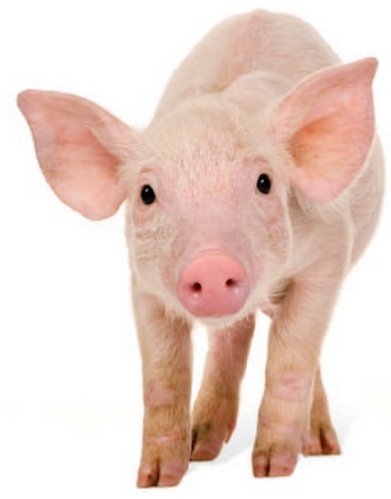

to chocolate milk. And the researchers had to add a 'start box' from which the piglets could enter the maze, because the piglets reacted badly when researchers carried them into the maze in initial trials.

Johnson's group plans to use the MRI and T-maze techniques to evaluate the effects of early life exposure to stressors on postnatal brain development. "This is the type of interesting question we can pursue now that the piglet model is in place," he said.

Monica Harrington

\section{SHEDDING LIGHT ON THE NEURAL NETWORKS UNDERLYING BEHAVIOR}

Optogenetics is a cutting edge technique being used by neuroscientists to determine the functions of specific brain cells. This technique involves inserting a light-sensitive gene into specific brain cells using a virus as a vector. The gene makes a protein called channelrhodopsin-2, which reacts to blue light by admitting ions into the brain cells, causing them to activate. In this way, researchers can activate individual brain cells by exposing them to light and then study the resulting physiological and behavioral effects.

So far, optogenetic methods have mostly been used in invertebrate and rodent studies. Previous studies using nonhuman primates only tested the technique on single isolated neurons and did not find any related behavioral effects. Wim Vanduffel and his team at Massachusetts General Hospital in Charlestown, MA, wanted to see if they could extend this research by studying the effect of optogenetically activating an entire network of cells.

First, the scientists scanned the brains of two rhesus macaques using functional magnetic resonance imaging (fMRI) while they performed a visual task. From the scans, they could determine which area of the brain was required for performing the task. Next, they inserted the light-reacting gene into multiple cells of this brain area and guided light-conducting needles into the same brain area so that light could be shone on the cells to boost their activity.

The researchers found that stimulating the brain cells with light improved the mental performance of the two macaques; the monkeys performed the visual task 10\% faster (Curr. Biol. published online 26 July 2012; doi:10.1016/j.cub.2012.07.023). These results show that pre-stimulating an area of the brain necessary for a task can lead to faster processing involved in performing the task. Vanduffel told New Scientist, "For the first time, we were able to change behavior in primates with our technique."

The team also observed optogenetically induced changes in fMRI activity in several specific brain networks during the task. The study also marks the first time that whole networks of cells were activated by optogenetic methods. This research opens up the possibility that the technique could be used to treat conditions in humans, such as epilepsy or Parkinson's disease, by activating populations of nonfunctioning brain cells. The researchers caution that much more work is to be done. "This is just proof of principle, and there's a very, very long way to go before we can start using it in humans," Vanduffel said. 